\title{
Jogo SB: estratégia lúdica de educação em saúde bucal para adolescentes na Amazônia
}

\author{
SB game: playful strategy for oral health education for \\ adolescents in the Amazon
}

\author{
Natália Lima Aguiar', Wallace Rafael Conde Barros', Ivam Freire da Silva Júnior², \\ Cyntia Maria Bino Sinimbú', Laysa Martins Barreto', Fabricio Moraes Pereira', \\ Liliane Silva do Nascimento' \\ 'Universidade Federal do Pará, Belém (PA), Brasil. \\ ${ }^{2}$ Universidade Federal de Pelotas, Pelotas (RS), Brasil.
}

\section{RESUMO}

Introdução: Na perspectiva de prevenção e promoção de saúde, a educação lúdica é utilizada como recurso capaz de produzir conhecimento crítico e despertar a análise sobre os hábitos que envolvem e conduzem ao estado de doença. Nesse contexto, o jogo passa a ser uma ferramenta ideal para aprendizagem, pois estimula o raciocínio lógico, a reflexão e a concentração para informações relevantes. Objetivo: Desenvolver e avaliar o efeito de uma metodologia inovadora de educação em saúde bucal para crianças e adolescentes na região amazônica. Método: Pesquisa ação desenvolvida por meio de um jogo de cartas intitulado Jogo SB (Saúde Bucal). Trata-se de um jogo investigativo no qual a finalidade é descobrir qual é o "Diagnóstico Confidencial" que consta de uma doença, uma causa e uma prevenção, que estão relacionados com temas de interesse de educação em saúde bucal para crianças e adolescentes. O jogo contem 69 cartas e regras, pode ser jogado por grupos de 2 a 4 jogadores. Para avaliação do jogo foi desenvolvido questionário aplicado antes e após o jogo. Resultados: Participaram da pesquisa 29 estudantes, sendo $15(51,7 \%)$ do sexo feminino e $14(48,3 \%)$ do sexo masculino, com idades entre 11 a 17 anos. Os resultados da observação revelaram que a participação e interesse dos adolescentes foram satisfatórios, a adesão ao jogo era imediata, demonstrando que técnicas alternativas de educação em saúde com jovens é atrativa e torna a incorporação do conhecimento algo dinâmico. Observou-se também um aumento médio de 16,08\% de respostas corretas no pós-jogo. Conclusão: A metodologia aplicada é adequada à educação em saúde bucal para crianças e adolescentes em qualquer ambiente, uma vez que, como jogo de cartas, independe de energia elétrica ou grandes recursos de infraestrutura. O jogo SB representa uma abordagem potencialmente viável para a absorção e ampliação do conhecimento, e permite ao jovem o empoderamento em autocuidado com a saúde bucal.

Recebido: Ago. 15, 2017 Aceito: Maio, 222019

\section{COMO CITAR ESTE ARTIGO}

Aguiar NL, Barros WRC, Silva Júnior IF, et al. Jogo SB: estratégia Júdicr para adol para adolescentes na Amazônia. Interdisciplinary Journal of Health Education. 2018 Jan-Dez;3(1-2):46-53. https://doi.org/10.4322/ijhe.2018.007

\section{CORRESPONDÊNCIA}

Fabricio Moraes Pereira

Programa de Pós-graduação em Saúde, Ambiente e Sociedade na Amazônia,

Universidade Federal do Pará

Rua Augusto Corrêa, 01, Campus Universitário do Guamá UFPA CEP $66075-110$ Belém (PA), Brasil

fabriciompbio@yahoo.com.br

\section{FONTE DE FINANCIAMENTO}

Nenhuma.

\section{CONFLITO DE INTERESSE}

Os autores declararam não

haver conflitos de interesse.

O estudo foi realizado na Universidade Federal do Pará, Belém (PA), Brasil.

Todos os autores leram e aprovam a versão final submetida ao Interdisciplinary Journal of

Health Education (IJHE).
PALAVRAS-CHAVE: Educação em saúde bucal. Adolescente. Jogos e brinquedos.

\begin{abstract}
Introduction: From the perspective of prevention and health promotion, playful education is used as a resource capable of producing critical knowledge and awaken the analysis of the habits that involve and lead to the disease state. In this context, the game becomes an ideal tool for learning, as it stimulates logical thinking, reflection and concentration for relevant information. Objective: To develop and evaluate the effect of an innovative methodology for oral health education for children and adolescents in the Amazon region. Method: Action research developed through a card game titled Game SB (Oral Health). This is an investigative game in which the aim is to discover what the "Diagnosis Confidential" which is contained in a disease, a cause and prevention that are related to topics of interest for oral health education for children and adolescents. The game contains 69 cards and rules, groups can be played by 2-4 players. To evaluation the game was developed questionnaire before and after the game. Results: 29 students participated in the survey, 15 (51.7\%) were females and 14 (48.3\%) males, aged 11-17 years. The results of observation revealed that the participation and interest of adolescents were high, and the involvement to the game was immediate, demonstrating that alternative techniques for health education with young people is attractive and makes the incorporation of knowledge something dynamic. We also observed an average increase of $16.08 \%$ of correct answers in the post-game. Conclusion: The methodology is suitable for oral health education for children and adolescents in any environment, since as the card game is independent of electricity or large infrastructure resources. The SB game represents a potentially viable approach for the absorption and expansion of knowledge, and allows the young man in self-empowerment with their oral health.
\end{abstract}

KEYWORDS: Oral health education. Teenager. Games and playthings. 


\section{Introdução}

A adolescência é vista como um período crítico de transição individual, onde os valores e opiniões transmitidos acerca do convívio social, cultural e familiar revertem a influência na construção de um perfil que porventura pode representar um comportamento de risco à saúde ${ }^{1,2}$. A interação entre fatores biológicos, socioculturais, econômicos e educacionais são relevantes na gênese das principais doenças bucais que acometem os adolescentes brasileiros.

Na perspectiva de prevenção e promoção em saúde, a educação lúdica, é utilizada como recurso capaz de produzir conhecimento crítico e despertar a reflexão sobre os hábitos que envolvem e conduzem ao estado de doença.

Segundo Barone et al. “ "Os jogos educativos permitem interação com o conteúdo, estimulando o aluno a pensar e tomar decisões a partir do momento que simula problemas e situações". Nesse contexto o jogo passa a ser uma ferramenta ideal para aprendizagem, pois estimula o raciocínio lógico, a reflexão e a concentração para informações relevantes. Pode ser utilizado com intuito de atrair e manter a atenção dos jogadores sobre temas que possivelmente gerem menos interesse em abordagens convencionais, do tipo palestras educativas.

No contexto da infância à adolescência, trabalhos demonstram algumas dificuldades na abordagem das práticas de educação em saúde, principalmente no que concerne à construção de conceitos, aprendizagem e mudança de comportamento. Ainda que haja informações importantes difundidas, faz-se necessária a utilização de práticas pedagógicas novas e diferenciadas na construção de conhecimentos acerca da prevenção e promoção de saúde $e^{4-7}$.

O conceito de educação em saúde também vai além da transmissão de informações, configurando combinações de experiências de aprendizagem delineadas com vistas a facilitar ações voluntárias conducentes à saúde . $^{8}$

Desta maneira, o uso de jogos educativos permite não apenas a introdução de novas informações dentro do contexto adolescente, mas sua análise e reflexão para tomadas de decisões. Mesmo que jogos funcionem por meio da competitividade e resulte em vencedores e perdedores, há um processo de construção de conhecimento durante esta trajetória que desafia os envolvidos a refletirem sobre saúde bucal.

Estudos têm demonstrado a preferência de crianças e adolescentes por jogos educativos, pois o método diferenciado também leva vantagem na absorção do conhecimento abordado. Ao brincar, os indivíduos praticam continuamente os processos complicados e estressantes do dia-a-dia, bem como adquirem maiores habilidades para se comunicar e alcançar relações satisfatórias com outras pessoas ${ }^{9,10}$.

Em saúde, estratégias lúdicas, como jogos, funcionam como ferramentas que facilitam a discussão de temas relevantes, como higiene ${ }^{11}$, hábitos saudáveis ${ }^{12}$, drogas ${ }^{13}$ e Doenças Sexualmente Transmissíveis ${ }^{14,15}$. Conforme descreve Garbin et al. ${ }^{16}$ quanto a estes dois últimos [...] tais assuntos são pouco discutidos entre os adolescentes, pois tendem a causar constrangimento e pouca liberdade de diálogo entre as famílias.

A Região Amazônica configura-se, por apresentar características peculiares e diversificadas, além de vários aspectos relacionados à elaboração de políticas públicas, é dotada de uma precariedade no acesso aos serviços de saúde, tanto por questões financeiras quanto geográficas ${ }^{17,18}$. De acordo com dados do Projeto SB Brasil $2010^{19}$, a região norte foi a única em que houve aumento do índice de cárie aos 12 anos em relação à pesquisa realizada em $2003^{20}$. Idade esta, que representa o início da adolescência, conforme Estatuto da Criança e do Adolescente ${ }^{21}$. A partir de determinantes que compõem este cenário, observa-se a necessidade de inovação na abordagem na Atenção Básica em saúde bucal voltada às crianças e adolescentes que vivem região. 
Com intuito de contribuir a outras práticas em saúde e para o incentivo ao uso de estratégias lúdicas e diversificadas para o fortalecimento de ações de prevenção, o objetivo deste estudo foi desenvolver e avaliar o efeito de um jogo de cartas como metodologia de educação em saúde bucal aplicadas para crianças e adolescentes na região amazônica.

\section{Metodologia}

Trata-se de uma pesquisa ação desenvolvida para contribuir na aquisição de conhecimentos sobre a saúde bucal de crianças e adolescentes de forma interativa e dinâmica.

Este trabalho teve aprovação CEP/ICS e segue todas as normativas de ética em pesquisa envolvendo seres humanos. As regras eram explicadas e a participação dos adolescentes seguiu os critérios estabelecidos na Resolução 466/12, de acordo com o parecer consubstanciado de número 1.659.973.

Desenvolveu-se um jogo de cartas intitulado "Jogo SB" com temas voltados à educação em saúde bucal. O jogo foi idealizado e produzido por estudantes do curso de Odontologia da Universidade Federal do Pará. A confecção das cartas foi feita por meio de parceria com estudante do curso de Artes da Universidade Federal do Pará.

Optou-se por um jogo de cartas devido a sua praticidade, pois pode ser aplicado em qualquer lugar, independente da utilização de grande logística ou de recursos, facilitando sua aplicação sob as características de algumas regiões da Amazônia. O jogo SB contém 69 cartas (Figura 1) e as regras descritivas. Pode ser jogado por grupos de 2 a 4 jogadores (Figura 2).Trata-se de um jogo investigativo no qual o objetivo é descobrir qual é o "Diagnóstico Confidencial" que consta de uma doença (cárie dentária; gengivite ou periodontite), uma causa ou consequência (mau hálito; boca seca; bactérias; placa ou inflamação) e uma prevenção (escovar os dentes; alimentação saudável; usar o fio dental; visitar o dentista regularmente ou não compartilhar escova dental). No inicio do jogo são removidas três Cartas Evidências, correspondentes a uma doença, uma causa e uma prevenção sem que os participantes vejam. Essas 3 cartas ficam embaixo da Carta "Diagnóstico Confidencial". O restante das Cartas Evidência é distribuído igualmente entre os jogadores. Cada participante recebe no inicio do jogo 13 Cartas Arquivos, correspondentes a todas as doenças, causas e prevenção mencionadas acima.
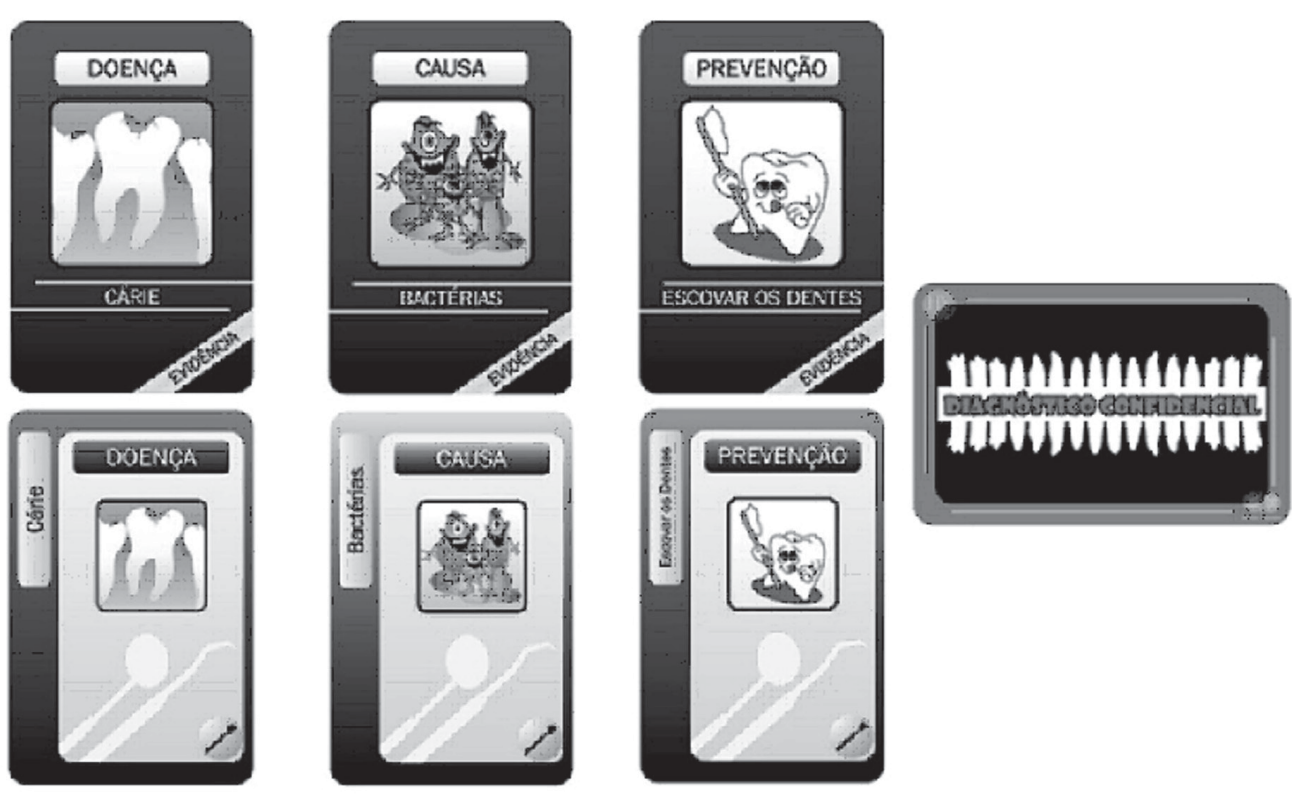

Figura 1. Representação gráfica das cartas do jogo (Fonte: produção dos autores). 


\section{ighe}

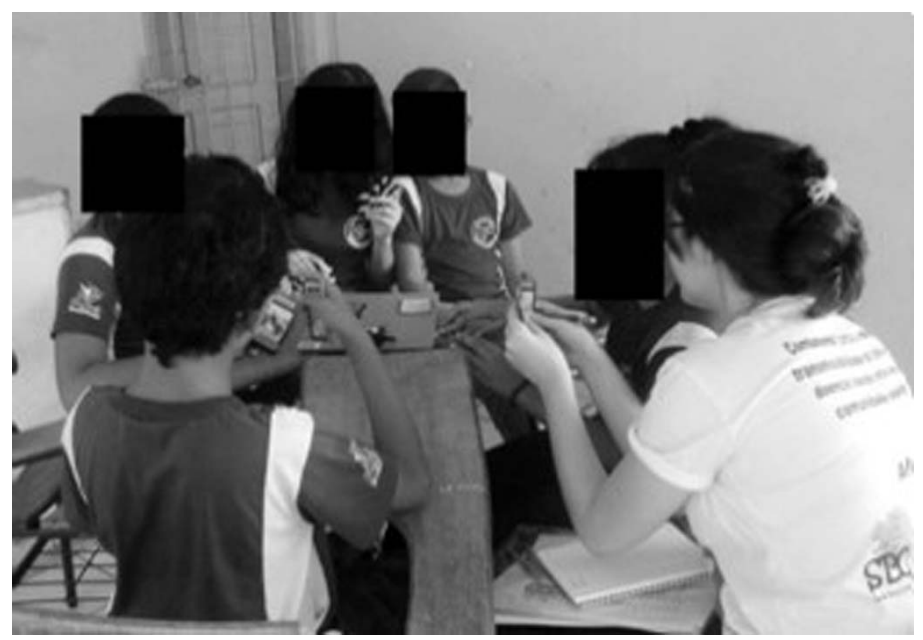

Figura 2. Grupo de escolares jogando o SB (Fonte: produção dos autores).

A partir da observação das cartas que são eliminadas durante a partida, o jogo segue até que algum dos participantes descubra o Diagnóstico e vença.

O jogo foi testado e analisado em estudo piloto através da observação direta, utilizando uma versão de teste com 20 escolares do Ensino Fundamental do município de Belém, Pará, Brasil. Neste momento verificou-se a aceitação por parte dos participantes em relação ao jogo e identificaram-se os ajustes necessários a serem realizados na apresentação das cartas.

Com base na análise dos dados observados conforme o jogo de teste, foi desenvolvida a versão final. Nesta fase, as regras foram alteradas, linguagem adaptada, e novos conteúdos incluídos. Avaliou-se também novo layout do jogo visual, produzido por especialistas gráficos.

Para avaliar o impacto educacional do jogo foi elaborado um questionário a ser aplicado antes e após o jogo, além da observação não participativa. O jogo então foi aplicado em outra Escola de Ensino Fundamental, também localizada no município de Belém, com 30 escolares.

Os escolares eram convidados a jogar uma partida e assim avaliados. Todas as partidas eram acompanhadas por algum aluno de odontologia. As observações eram registradas em diário de campo de pesquisa. Os dados foram coletados em instrumento próprio e analisados descritivamente.

\section{Resultados e discussão}

Do total de participantes, um foi excluído da pesquisa por não responder todas as questões do questionário, permanecendo então 29 escolares participantes, dos quais $51,7 \%$ (15) eram do sexo feminino e $48,3 \%$ (14) do sexo masculino, com idades entre 11 a 17 anos. O jogo foi aplicado durante o período de dois meses na escola, nos intervalos das aulas ou em aulas vagas.

Em relação ao conceito de cárie no momento inicial do jogo, perguntou-se "O que é a cárie dentária?" A grande maioria dos participantes (82,7\%) marcou erroneamente a alternativa que refere à cárie como uma bactéria. A cárie dentária é uma doença multifatorial, infecciosa, transmissível e dieta dependente, que produz desmineralização das estruturas dentárias, podendo afetar esmalte, dentina, cemento e eventualmente chegar a comprometer a polpa ${ }^{22,23}$. Esse resultado diverge do de Flores e Drehmer ${ }^{24}$, que mostra que a maioria dos participantes da pesquisa reconhece a cárie como doença, considerada um problema comum aceitado como normal e decorrente de situações de desequilíbrio. Após aplicação do jogo e sua análise, notou-se que a representação simbólica da doença cárie melhorou em 20,7\%, conforme Tabela 1, 
Tabela 1. Distribuição das respostas dos escolares participantes do jogo SB em dois momentos da avaliação antes e após, Belém, 2013.

\begin{tabular}{|c|c|c|c|c|}
\hline \multirow{2}{*}{ O que é cárie dentária? } & \multicolumn{2}{|c|}{ Pré Jogo } & \multicolumn{2}{|c|}{ Pós Jogo } \\
\hline & $\mathbf{N}$ & $\%$ & $\mathbf{n}$ & $\%$ \\
\hline Uma bactéria no dente & 24 & 82,7 & 19 & 65,5 \\
\hline Uma doença do dente & 2 & 6,9 & 8 & 27,6 \\
\hline Um buraco no dente & 3 & 10,4 & 2 & 6,9 \\
\hline A periodontite é uma doença que prejudica gengiva e osso? & $\mathbf{N}$ & $\%$ & $\mathbf{n}$ & $\%$ \\
\hline Sim & 6 & 20,7 & 14 & 48,2 \\
\hline Não & 4 & 13,7 & 3 & 10,4 \\
\hline Não Sei & 19 & 65,6 & 1 & 41,4 \\
\hline O mau hálito pode ser provocado por cárie e por gengivite? & $\mathbf{N}$ & $\%$ & $\mathbf{n}$ & $\%$ \\
\hline Sim & 18 & 62 & 20 & 68,9 \\
\hline Não & 6 & 20,7 & 6 & 20,7 \\
\hline Não Sei & 5 & 17,3 & 3 & 10,4 \\
\hline Quando a boca tem pouca saliva (boca seca) pode causar cárie? & $\mathbf{N}$ & $\%$ & $\mathbf{n}$ & $\%$ \\
\hline Sim & 6 & 20,7 & 11 & 37,9 \\
\hline Não & 8 & 27,6 & 7 & 24,2 \\
\hline Não Sei & 15 & 51,7 & 11 & 37,9 \\
\hline A perda do dente pode ser por cárie dentária e por doenças da gengiva? & $\mathbf{N}$ & $\%$ & n & $\%$ \\
\hline Sim & 18 & 62 & 20 & 68,9 \\
\hline Não & 6 & 20,7 & 5 & 17,3 \\
\hline Não Sei & 5 & 17,1 & 4 & 13,8 \\
\hline
\end{tabular}

demonstrando que o jogo proporcionou aumento no entendimento sobre cárie dentária pelos participantes.

Sobre as doenças periodontais, questionou-se "A periodontite é uma doença que prejudica gengiva e osso?". Tal questão foi levantada pela prevalência descrita no SB Brasil 2010 do Índice Periodontal Comunitário (CPI) em adolescente onde a presença de cálculo foi a pior condição periodontal observada, seguida do sangramento. $\mathrm{O}$ estudo ainda afirma que os problemas periodontais avaliados pelo CPI tendem a aumentar com a idade ${ }^{19}$. Obteve-se no pós-jogo um aumento de $27,5 \%$ nas respostas positivas, conforme demonstra a Tabela 1, indicando a atuação positiva do jogo na educação sobre doenças periodontais.

É válido ressaltar que os adolescentes caracterizam uma população com grande potencial para a prevenção de doenças, sobretudo a cárie e doença periodontal, pois sua sistematização cognitiva está atrelada à regularidade de informações e percepções de saúde geral e bucal que contribuem para a adesão de hábitos saudáveis s, $26^{26}$.

A halitose representa um fenômeno que acompanha o homem na sua trajetória social-histórica e pode ser negativa para a autoimagem do indivíduo, impactando na confiança e causando evitação social. Para Emmerich e Castiel ${ }^{27}$, existe uma estigmatização do mal-estar social inerente aos maus odores bucais. Essa estigmatização desnecessária da questão está subliminarmente associada à tentativa biopolítica de tornar os sujeitos mais sociáveis e, também, à comercialização de "mercadorias" para melhor aceitação no mercado de trabalho, à autoaceitação e ao maior "conforto" social. Sobre a halitose na adolescência foi feita a pergunta: "O mau hálito pode ser provocado por cárie e por gengivite?". Notou-se que no pós-jogo a representação simbólica do 
mau hálito provocado por cárie e gengivite melhorou em 6,9\% (Tabela 1), indicando a melhora na taxa de resposta sobre o tema tão estigmatizante na adolescência.

Quanto à abordagem referente à saliva: “Quando a boca tem pouca saliva (boca seca) pode causar cárie?" obteve-se um aumento de 17,2\% no pós-jogo (Tabela 1), em que a doença cárie também possui etiologia associada à baixa salivação, este aumento indica que o jogo atingiu seu objetivo quanto à educação sobre a relação de cárie e salivação. Segundo Farghaly et al. ${ }^{28}$, a saliva desempenha papéis importantes na defesa contra a cárie dentária, sua ação mecânica de limpeza diminui a disponibilidade de substratos essenciais para o crescimento da placa bacteriana cariogênica. A capacidade tampão, a supersaturação de cálcio e fosfato e a manutenção de eletrólitos na forma iônica aumentam a remineralização dentária e impedem o processo de desmineralização.

Sobre a perda dentária os participantes foram abordados da seguinte forma: "A perda do dente pode ser por cárie dentária e por doenças da gengiva?" Percebe-se que após o jogo a representação simbólica das causas da perda dentária melhorou em 6,9\% (Tabela 1). A extração dos dentes é considerada como uma decorrência do agravamento das doenças bucais mais predominantes, entre elas a cárie e doença periodontal. Entretanto, na prática dá-se como solução definitiva para dor, sendo motivada principalmente pela falta de acesso ao serviço e à condição socioeconômica ${ }^{29}$.

Os dados obtidos nos resultados da pesquisa demonstram as contribuições do jogo como ferramenta de educação em saúde bucal para crianças e adolescentes. Inicialmente, os alunos ficaram confusos com as regras e o envolvimento de um preceptor foi exigido. Durante a segunda partida, os alunos ficaram mais independentes e interessados em jogar. Esta independência pode ser resultado da facilitação da autonomia, já que a estratégia de educação em saúde utilizada neste trabalho possui relevância social e direciona maior entendimento acerca de processos de saúde bucal ${ }^{30}$.

A diferença entre pré e pós-jogo na pontuação em conhecimentos/competências sugere a capacidade dos adolescentes de aprender comportamentos de autocuidado em um tempo relativamente breve, e o aumento do nível de confiança para praticar os novos comportamentos aprendidos em suas vidas diárias.

No entanto, uma limitação deste estudo foi que a avaliação do jogo de cartas foi realizada uma única vez após uma partida, portanto a aquisição de conhecimentos foi pontual. Não foi possível medir os resultados em longo prazo ou confirmar que a mudança de comportamento poderia ocorrer após a intervenção educativa. Estima-se que com a repetição de partidas o conhecimento será cada vez mais reconhecido, possibilitando ferramentas para a mudança de comportamento e criação de agentes multiplicadores do cuidado em saúde bucal.

O formato simples do jogo de cartas é útil e facilmente adaptado não só para as escolas, mas também para a casa, comunidade e outros contextos não formais.

\section{Conclusões}

A estratégia inovadora do jogo SB mostrou-se capaz de promover educação em saúde bucal para os adolescentes de maneira atrativa e informal, permite a circulação de informações relevantes à saúde bucal do adolescente. Ressalta-se que o jogo permite ao jovem o empoderamento potencial em autocuidado, criando condições para que ele seja um agente ativo na sua saúde e multiplicador de informações em saúde na sua comunidade.

\section{Referências}

1. Filgueira ACG, Machado FCA, Amaral BA, Lima KC, Assunção IV. Saúde bucal de adolescentes escolares. Rev Holos. 2016;32(1):161-72. http://dx.doi.org/10.15628/holos.2016.3577.

2. Chimeli IV. Adolescências e saúde: um estudo sobre comportamentos de risco [monografia]. Belo Horizonte: Fundação Oswaldo Cruz; 2015. 
3. Barone DAC, Figueiredo MC, Garcia M, Oliveira R. O uso de um jogo eletrônico sobre saúde bucal para escolares da zona rural. In: Anais do IX Congresso Latino-Americano Interdisciplinar Orientado ao Adolescente [recurso eletrônico]; 2015 jun 4-16; Porto Alegre, RS. Porto Alegre (RS): Universidade Federal do Rio Grande do Sul; 2015.

4. Michie S, West R. Behaviour change theory and evidence: a presentation to government. Health Psychol Rev. 2013;7(1):122. http://dx.doi.org/10.1080/17437199.2011.649445.

5. Perim CM, Giannella TR, Struchiner M. Análise do uso de um jogo para educação em saúde com adolescentes. Ensino, Saúde e Ambiente. 2014;7(1):1-12. Edição especial.

6. Aljafari A, Gallagher JE, Hosey MT. Failure on all fronts: general dental practioners' views on promoting oral health in high caries risk children- a qualitative study. BMC Oral Health. 2015;15(45):45. http://dx.doi.org/10.1186/s12903-015-0032-8. PMid:25888427.

7. Gonçalves LFF, Faria DSA, Batista ES, Ferreira SR, Assis SM. Promoção de saúde com adolescentes em ambiente escolar: relato de experiência. SANARE. 2016;15(2):160-7.

8. Figueiredo MC, Garcia M, Barone DAC, Oliveira R, Lurmmerz G. Gameficação em saúde bucal: experiência com escolares de zona rural. Revista da ABENO. 2015;15(3):98-108. http://dx.doi.org/10.30979/rev.abeno.v15i3.195.

9. Araújo NMS, Ribeiro FR, Santos SF. Jogos pedagógicos e responsividade: ludicidade, compreensão leitora e aprendizagem. Bakhtiniana: Rev Estud Discurso. 2012;7(1):4-23. http://dx.doi.org/10.1590/S2176-45732012000100002.

10. Adade M, Monteiro S. Educação sobre drogas: uma proposta orientada pela redução de danos. Educ Pesqui. 2014;40(1):21530. http://dx.doi.org/10.1590/S1517-97022013005000009.

11. Coscrato G, Pina JC, Mello DF. Utilização de atividades lúdicas na educação em saúde: uma revisão integrativa da literatura. Acta Paul Enferm. 2010;23(2):257-63. http://dx.doi.org/10.1590/S0103-21002010000200017.

12. Souza EP, Ferreira LR, Borges LJC. Jogo digital como instrumento de reflexão sobre hábitos alimentares e prática de atividade física na adolescência. In: Anais do XII Seminário de SJEEC; 2017; Bahia (BA). Vitória da Conquista (BA): Centro Juvenil de Ciência e Cultura de Vitória da Conquista; 2017.

13. Damasceno EF, Nardi PA, Silva AA, Lopes LLFB, Fernandes AM. Um serious game como estratégia na promoção da saúde no combate ao uso de drogas. J Bras Tele. 2016;4(2):237-45.

14. Oliveira RNG, Gessner R, Souza V, Fonseca RMGS. Limites e possibilidades de um jogo online para a construção de conhecimento de adolescentes sobre a sexualidade. Rev Cien Saude Colet. 2016;21(8):2383-92. http://dx.doi.org/10.1590/141381232015218.04572016. PMid:27557012.

15. Souza V, Gazzinelli MF, Soares AN, Fernandes MM, Oliveira RNG, Fonseca RMGS. The game as strategy for approach to sexuality with adolescents: theoretical-methodological reflections. Rev Bras Enferm. 2017;70(2):376-83. http://dx.doi. org/10.1590/0034-7167-2016-0043. PMid:28403303.

16. Garbin CS, Lima DP, Dossi AP, Arcieri RM, Rovida TAS. Percepção de adolescentes em relação a doenças sexualmente transmissíveis e métodos contraceptivos. DST - J Bras Doenças Sex Transm. 2010;22(2):60-3.

17. Teixeira E, Siqueira AA, Silva JP, Lavor LC. Cuidados com a saúde da criança e validação de uma tecnologia educativa para famílias ribeirinhas. Rev Bras Enferm. 2011;64(6):1003-9. http://dx.doi.org/10.1590/S0034-71672011000600003. PMid:22664596.

18. Rodrigues PCO, Ignotti E, Hancon SS. Características socioeconômicas, demográficas e de saúde de escolares residentes em duas comunidades da Amazônia meridional brasileira. Revista Ciência e Estudos Acadêmicos de Medicina. 2016;6:10-23.

19. Brasil. Ministério da Saúde. Projeto SB Brasil 2010: Pesquisa Nacional de Saúde Bucal - Resultados Principais. Brasília: Ministério da Saúde; 2011.

20. Brasil. Ministério da Saúde. Projeto SB Brasil 2003: Pesquisa Nacional de Saúde Bucal - Resultados Principais. Brasília: Ministério da Saúde; 2004.

21. Brasil. Presidência da República, Subchefia para Assuntos Jurídicos. Lei no 8.069 , de 13 de julho de 1990, e legislação correlata. Estatuto da Criança e do Adolescente [Internet]. Brasília, DF, 2012 [citado em 2017 Jul 20]. Disponível em: http:// www.planalto.gov.br/ccivil_03/leis/L8069.htm

22. Keyes PH. Presentand future measures for dental caries control. J Am Dent Assoc. 1969;79(6):1395-404. http://dx.doi. org/10.14219/jada.archive.1969.0037. PMid:4902885.

23. Stookey GK. The effect of saliva on dental caries. J Am Dent Assoc. 2008;139(Suppl):11S-7S. http://dx.doi.org/10.14219/ jada.archive.2008.0347. PMid:18595200.

24. Flores EMTL, Drehmer TM. Conhecimentos, percepções, comportamentos e representações de saúde e doença bucal dos adolescentes de escolas públicas de dois bairros de Porto Alegre. Cien Saude Colet. 2003;8(3):743-52. http://dx.doi. org/10.1590/S1413-81232003000300008.

25. Oliveira RCN, Souza JGS, Oliveira CC, et al. Acesso a informações sobre como evitar problemas bucais entre escolares da Rede Pública de Ensino. Ciênc \& Saúde Coletiva. 2015;20(1):85-94. http://dx.doi.org/10.1590/1413-81232014201.00032014.

26. Marín C, Papadopol PM, Bottan ER, Orcina BF. Percepção e informação sobre saúde bucal: estudo com adolescentes de uma escola pública. Revista Saúde e Pesquisa [Internet] 2016 [citado em 2017 Jul 7];9(3):499-506. Disponível em: http:// periodicos.unicesumar.edu.br/index.php/saudpesq/article/view/5417

27. Emmerich A, Castiel LD. Mais humano que um humano: a halitose como emblema da patologização odontológica. Cien Saude Colet. 2012;17(1):89-98. http://dx.doi.org/10.1590/S1413-81232012000100012. PMid:22218543.

28. Farghaly JG, Fachin LV, Otton R, Guaré RO, Leite MF. Efeito do gênero (masculino e feminino) sobre a cárie dentária e parâmetros salivares de crianças. Pesq Bras Odontoped Clin Integrada. 2013;13(1):11-5. http://dx.doi.org/10.4034/ PBOCI.2013.131.02.

29. Costa SM, Abreu MHNG, Vasconcelos M, Lima RCGS, Verdi M, Ferreira EF. Desigualdades na distribuição da cárie dentária no Brasil: uma abordagem bioética. Cien Saude Colet. 2013;18(2):461-70. http://dx.doi.org/10.1590/S141381232013000200017. PMid:23358771.

30. Fadel CB, Bordin D, Langoski JE. A educação como prática viabilizadora da saúde bucal. J Health Sci Inst. 2013;31(2):136-40. 


\section{ijhe}

\section{Contribuição dos autores}

Natália Lima Aguiar participou de todas as etapas da pesquisa e da confecção do manuscrito; Wallace Rafael Conde Barros e Ivam Freire da Silva Júnior participaram da elaboração do jogo, aplicação com os escolares, coleta de dados e confecção do manuscrito; Cyntia Maria Bino Sinimbú e Laysa Martins Barreto participaram da aplicação com os escolares, coleta de dados e confecção do manuscrito; Fabricio Moraes Pereira participou da análise de dados, acompanhamento, orientação do estudo, confecção, elaboração e revisão do manuscrito; Liliane Silva do Nascimento participou na concepção inicial, acompanhamento, orientação do estudo, elaboração e revisão do manuscrito. 